\title{
Custo da implantação e utilização de dois métodos de identificação de bovinos leiteiros
}

\author{
Marcos Aurélio Lopes ${ }^{1}$, Marilane das Dores Silva², Andréia Alves Demeu ${ }^{3}$, Débora Ribeiro Gomide ${ }^{4}$, \\ Fábio Raphael Pascoti Bruhn
}

\section{RESUMO}

Neste estudo, objetivou-se avaliar o custo da implantação e utilização de dois métodos de identificação de bovinos, o do brinco auricular convencional e o da arruela auricular eletrônica, além de discutir as vantagens e limitações de cada um. Foram anotadas todas as despesas operacionais referentes à identificação e, dentro de cada método, foram mensurados os tempos de contenção do animal, de implantação do identificador, de leitura do brinco, de digitação e de transferência de dados. O custo da identificação da arruela auricular eletrônica foi mais elevado, principalmente em função da aquisição dos equipamentos e dispositivos de identificação. Entretanto, verificou-se maior confiabilidade das informações registradas, já que o brinco auricular convencional, apesar do menor custo, apresentou erros na transcrição dos dados. Assim, cabe a cada produtor e, ou, técnico definir qual deve ser o método de identificação adequado ao seu sistema de produção, considerando não apenas os aspectos financeiros, mas também as limitações e benefícios de cada um.

Palavras-chave: automação, bovinocultura, identificação eletrônica.

\section{ABSTRACT}

\section{Cost of the implantation and utilization of two methods of identification of dairy cattle}

This study aimed to evaluate the cost of the implantation of two methods of cattle identification, namely conventional earring and electronic ear tag, in addition to discussing both the advantages and limitations of each of them. All the operational expenses concerning to the identification were recorded and, for each method, the times of holding of the animal, the implantation of the identifier, the earring reading, typing and data transfer were surveyed. The cost of the identification by using the electronic tag was the highest, mainly the purchase of the equipment and identification device. Nevertheless, there was increased reliability of the recorded information, since the conventional earring in spite of having lower cost, induced errors in data transcription. Therefore, it is up to the farmers to decide which method should be suitable for their production system, taking into account not only financial issues but also the limitations and benefits of each method.

Key words: automation, cattle, electronic identification.

\footnotetext{
Recebido para publicação em 05/03/2012 e aprovado em 03/06/2013.

${ }^{1}$ Licenciado em Ciências Agrícolas, Doutor. Departamento de Medicina Veterinária, Universidade Federal de Lavras, Campus Universitário, s/n, Caixa Postal 3037, 37200-000, Lavras, Minas Gerais, Brasil. malopes@dmv.ufla.br (autor para correspondência).

${ }^{2}$ Graduanda em Zootecnia. Departamento de Medicina Veterinária, Universidade Federal de Lavras, Campus Universitário, s/n, Caixa Postal 3037, 37200-000, Lavras, Minas Gerais, Brasil. marylanezoo@yahoo.com.br

${ }_{3}^{3}$ Zootecnista. Departamento de Medicina Veterinária, Universidade Federal de Lavras, Campus Universitário, s/n, Caixa Postal 3037, 37200-000, Lavras, Minas Gerais, Brasil. andreiademeu@hotmail.com

${ }^{4}$ Médica Veterinária. Empresa de Pesquisa Agropecuária de Minas Gerais - EPAMIG, Rodovia Três Pontas, Santana da Vargem, Km 06, Caixa Postal 91, 37190-000, Zona Rural, Três Pontas, Minas Gerais, Brasil. deboragomide@epamig.br

${ }^{5}$ Médico Veterinário. Departamento de Medicina Veterinária, Universidade Federal de Lavras, Campus Universitário, s/n, Caixa Postal 3037, 37200-000, Lavras, Minas Gerais, Brasil. fabio_rpb@yahoo.com.br
} 


\section{INTRODUÇÃO}

Os sistemas de identificação animal são ferramentas importantes na administração rural, que possibilitam individualizar os animais do plantel e, a partir daí, acompanhar seu desenvolvimento e seus índices zootécnicos. Esses sistemas vão desde o uso de nomes, em pequenos rebanhos, até chips com códigos eletrônicos. Com os animais individualizados, tornam-se mais fáceis e ágeis os procedimentos nas diferentes fases de manejo, como descarte, desmama, reposição e outros (Marsão \& Gonçalves, 2009).

A identificação segura dos animais é a base para todas as funções do sistema de manejo, que resultam em progressos zootécnicos, controle e economia da produção (Lopes, 1997). No Brasil, o brinco auricular convencional é um dos principais métodos de identificação adotados. Existem vários tipos no mercado, que variam em preços, cores e formatos, com código de barras, numeração impressa ou, em branco, dentre outros.

Nos últimos anos, a necessidade que as propriedades agropecuárias têm apresentado em relação à melhoria contínua nos seus níveis de qualidade, produtividade e competitividade, tem ocasionado o desenvolvimento de soluções inovadoras, que envolvem níveis diferenciados de automação (Banzato, 2002). Segundo a Embrapa (1996), o panorama mundial permite vislumbrar, para um futuro próximo, dependência inevitável da automação nos processos de produção. A automação poderá auxiliar profundamente na sustentabilidade, tanto dos processos de produção, quanto no desenvolvimento econômico e social.

Vários pesquisadores têm-se preocupado em estudar alguns aspectos da identificação, principalmente os diferentes métodos utilizados (Jardim, 1973; Lopes \& Vieira, 1998; Curto, 2002). De acordo com Lopes (1997), na medida em que os rebanhos tornam-se maiores e mais bem manejados, a identificação dos animais torna-se um importante problema. Assim, a necessidade de um método de identificação permanente, sem danos para o animal e facilmente legível, preciso, rápido, sem erros, é notória. Com o avanço da tecnologia, métodos mais aprimorados vêm sendo desenvolvidos, dentre eles a identificação eletrônica. Por ainda constituir novidade, tem sido objeto de diversos estudos. Curto (2002), em ampla revisão da literatura, abordou aspectos da tecnologia em si, e da sua interação com sistemas informatizados, bem como dos sistemas disponíveis no mercado. Esse último aspecto também foi amplamente discutido por Curto et al. (1997), Lopes (1997), Lopes (2003) e Lopes (2005).

No entanto, esses autores não abordaram os impactos econômicos, nem mesmo o custo/benefício de cada um dos diferentes métodos, muito embora essa tenha sido, e continua sendo, uma das principais preocupações dos pecuaristas. Um dos raros estudos abordando aspectos econômicos foi realizado por Lopes et al. (2008), que analisaram a viabilidade econômica da implantação de um sistema de rastreabilidade bovina, utilizando apenas brinco auricular convencional e botton.

Considerando-se que a legislação permite a utilização de diferentes métodos de identificação, que cada um possui preços diferenciados e apresenta vantagens e limitações, que se verifica também pela escassez de pesquisas sobre a questão, principalmente no aspecto econômico, torna-se evidente a necessidade de mais estudos. Diante disso, objetivou-se avaliar o custo da implantação e utilização de dois métodos de identificação de bovinos, o do brinco auricular convencional e o da arruela auricular eletrônica, bem como discutir algumas vantagens e limitações de cada um, visando a fornecer informações que subsidiem técnicos e pecuaristas na escolha do método mais adequado para identificar os bovinos.

\section{MATERIAL E MÉTODOS}

A pesquisa foi realizada de julho a novembro de 2009, na fazenda experimental da Epamig (Empresa de Pesquisa Agropecuária de Minas Gerais), no município de Três Pontas, região sul de Minas Gerais. Foram comparados dois métodos de identificação: o do brinco auricular convencional e o da arruela auricular eletrônica. Em cada método, foram mensurados os tempos de contenção do animal, de implantação dos dispositivos de identificação, de leitura e de transferência dos dados para um microcomputador. Além disso, foi analisado o custo dos brincos, bem como os erros na transcrição de dados, visando à análise do custo e à viabilidade de utilização. Os tempos, em segundos, foram mensurados, utilizando-se cronômetros, por dois pesquisadores.

Os dois métodos de identificação foram implantados em animais das raças holandesa e mestiços (gir x holandês), com idade entre 13 dias e 18 meses. A quantidade total de animais identificados foi de 36 , sendo utilizado o brinco auricular convencional, em 13, e, a arruela auricular eletrônica, em 23 animais. Além dos dispositivos de identificação, utilizaram-se ainda repelente, unguento e alicate universal para aplicação de brincos.

A identificação, com uso do brinco auricular convencional e da arruela auricular eletrônica, dos bezerros com até 91 dias de idade ocorreu no bezerreiro, coberto por sombrite, onde os animais estavam presos em correntes. A cronometragem do tempo para colocação dos dispositivos começava no momento em que duas pessoas iniciaram os preparativos, ou seja, enquanto uma encaixava o dispositivo no alicate, a outra realizava a contenção do animal. Ao ser encaixado no alicate, o dispositivo recebia uma pequena quantidade de unguento no perfurador 
auricular e era então aplicado na orelha esquerda do animal. Após a implantação, aplicava-se repelente, após o que finalizava-se a cronometragem do tempo. A implantação dos dispositivos de identificação dos animais com idade superior a 91 dias foi realizada em um tronco de contenção, por duas pessoas: uma prendia a cabeça do animal e a outra aplicava o dispositivo de identificação.

A leitura do brinco auricular convencional ocorreu no bezerreiro, sendo feita por duas pessoas. A cronometragem do tempo de leitura iniciava-se quando o pesquisador aproximava-se do animal e lia, em voz alta, o número, que uma segunda pessoa anotava, finalizando-se a cronometragem com a inscrição do número lido, em caderneta de campo.

Quanto aos animais conduzidos até o brete para colocação do brinco convencional, a leitura do seu dispositivo de identificação foi feita no mesmo local. A cronometragem do tempo iniciava-se com a entrada do animal na seringa, conduzido por duas pessoas, e finalizou-se com o fechamento do brete. A cronometragem do tempo de leitura era iniciada com o pesquisador aproximando-se do animal, lendo o número em voz alta, seguindo-se de sua anotação pelo outro pesquisador.

A leitura da arruela auricular eletrônica ocorreu somente no brete, sendo mensurado o tempo necessário para o acoplamento do cabo de leitura ao leitor, assim como o tempo de entrada dos animais na seringa até o fechamento do brete. $\mathrm{O}$ tempo de leitura era cronometrado a partir do momento que o pesquisador aproximava-se do animal; estendia o leitor em direção à arruela auricular eletrônica, pressionando o botão para "ler" as informações, até que, ao final da leitura, indicado pelo som de um bip, os cronômetros eram parados.

Os dados dos animais, lidos e armazenados no leitor, eram transferidos para um software (download dos dados) e o tempo necessário para essa atividade era contabilizado. A cronometragem do tempo iniciava-se com a montagem do equipamento. Ligava-se o leitor e era acoplado o cabo serial; posteriormente, conectando-se a outra extremidade do cabo ao computador. Acessava-se então o software. No leitor, selecionava-se a opção configuração; em seguida a comunicação, iniciando-se o download dos dados. Salvado o arquivo, desmontava-se o equipamento e, ao final dessa ação, desligavam-se os cronômetros. O tempo mensurado era registrado.

Os números dos brincos auriculares convencionais, lidos e anotados em caderneta de campo, eram digitados em uma planilha eletrônica, desenvolvida no Excel $^{\circledR}$. Os tempos gastos na digitação e na conferência dos dados eram cronometrados por dois pesquisadores. Avaliaramse ainda erros nas digitações.

A construção do banco de dados e as análises estatísticas foram realizadas no software SPSS $17.0^{\circledR}(2011)$. Foi realizada a descrição dos dados obtidos no campo. Além disso, buscou-se verificar a ocorrência de diferenças nos tempos gastos ao se utilizar a arruela auricular eletrônica e o brinco auricular convencional. Assim, foi aplicado o teste de normalidade de Kolmogorov-Smirnov, e, depois de verificado que somente a variável tempo para implantação do brinco apresentava distribuição normal, foi realizado o teste t de Student nessa variável e, o teste MannWhitney, nas variáveis tempo de contenção e tempo para a leitura da informação no animal. Além disso, foram comparadas as médias de tempo gasto nessas três variáveis em função da idade dos animais. Assim, a amostra foi dividida em animais com: 1) até 91 dias; 2) de 92 a 375 dias; e 3) mais de 375 de idade. As variáveis tempo no brete de contenção e tempo para a leitura da informação no animal foram submetidas ao teste de Kruskal-Wallis, e, posteriormente, ao teste de Mann-Whitney entre os grupos por idade, visando a identificar onde estavam as diferenças encontradas. A variável tempo para implantação foi submetida ao teste de Anova. O teste de correlação de Spearman e Pearson também foi aplicado entre, respectivamente, as variáveis tempo de contenção/tempo de leitura e tempo de implantação dos dispositivos de identificação e idade dos animais avaliados. Foi usada significância mínima de 5\%, em todos os métodos estatísticos.

$\mathrm{Na}$ análise estatística foram desconsiderados três tempos de implantação do brinco auricular convencional e oito tempos de implantação da arruela auricular eletrônica, por serem discrepantes em relação à média dos tempos e, caso fossem considerados, superestimarem os resultados dessa análise.

Para a análise dos custos dos dois métodos, foram registradas todas as despesas operacionais para sua implantação e o custo da leitura dos dispositivos de identificação, que foi contabilizado pelo valor da mão de obra. Foram quantificados os custos variáveis, ou seja, aqueles que variam em função da quantidade de animais, e os custos fixos, aqueles que se referem à depreciação dos equipamentos utilizados na identificação (Lopes \& Santos, 2007), considerando-se que esses equipamentos serão utilizados em vários ciclos produtivos. A depreciação foi calculada pelo método linear (Hoffmann et al., 1981).

\section{RESULTADOS E DISCUSSÃO}

Não foram verificadas diferenças entre os tempos médios da implantação da arruela auricular eletrônica e a do brinco auricular convencional, da contenção dos animais e da leitura dos brincos (Tabela 1). No que diz respeito à contenção e implantação do dispositivo de identificação, isso era esperado, pois os procedimentos foram os mesmos. Quanto ao tempo de leitura, o fato de não se ter 
verificado diferença $(\mathrm{P}=0,135)$, o que não era esperado, pode ser explicado por ter sido considerado o tempo necessário para a montagem e preparo do equipamento eletrônico. Esse tempo foi somado ao tempo da leitura, propriamente dita, e dividido pela pequena quantidade de animais. À medida que a quantidade de animais a serem "lidos" aumenta, o tempo de montagem e preparo do equipamento eletrônico será "diluído", contribuindo para aumentar a diferença, justificando a utilização da automação. Embora não se tenha constatado diferenças nos tempos de leitura dos dispositivos de identificação, a diferença no tempo de leitura do brinco auricular convencional chega a ser, numericamente, $30 \%$ superior ao tempo despendido para a leitura da arruela eletrônica. Essa diferença, do ponto de vista da gestão, é relevante, pois quanto menor o tempo gasto na leitura, menor será o tempo do uso da mão de obra para esse serviço, reduzindo-se o custo. O uso do bastão leitor favorece a leitura das arruelas eletrônicas, pois essa pode ser realizada a uma distância de $20 \mathrm{~cm}$; além disso, a percepção visual do pesquisador, ao ler o brinco auricular convencional, pode influenciar, aumentando o tempo de leitura, bem como a movimentação do animal durante o procedimento. Além do que, segundo Lopes (1997), alguns problemas podem surgir durante a leitura dos brincos convencionais, por causa da abrasão dos caracteres e da adesão de sujeiras. Nesta pesquisa, isso não ocorreu, contribuindo para reduzir o tempo de leitura do brinco auricular convencional.

Observou-se correlação positiva entre a idade dos animais e o tempo de leitura $(0,256 ; \mathrm{P}<0,01)$, ou seja, à medida que a idade do animal aumenta, necessita-se de um tempo de leitura maior; e, negativa, com o tempo de contenção $(-0,261 ; \mathrm{P}<0,01)$, ou seja, à medida que aumenta a idade do animal, diminui o tempo gasto para conter os animais maiores (Tabelas 2 e 3). Tendo em vista que os animais com mais de 91 dias foram contidos no brete, justifica-se o menor tempo na contenção, e os animais, à medida que crescem, tornam-se mais ariscos, dificultando a leitura dos dispositivos de identificação. O mesmo foi verificado ao se considerar apenas o dispositivo con- vencional de identificação: correlação positiva $(0,375$; $\mathrm{P}<0,01)$, entre a idade dos animais e o tempo de leitura, $\mathrm{e}$ negativa $(-0,339 ; \mathrm{P}<0,01)$, entre a idade e o tempo de contenção. Não foram observadas correlações significativas $(\mathrm{P}>0,05)$ para a variável tempo de implantação, ao se considerar somente os animais com brinco auricular convencional. No entanto, é importante salientar que, neste estudo, a maioria das correlações encontradas, apesar de estatisticamente significativas, é de fraca intensidade (menor que 0,3 , ou seja, o tempo cresce ou decresce de maneira pouco proporcional às diferentes idades dos animais.

Não houve diferenças significativas para as variáveis tempo de contenção e implantação entre os dispositivos de identificação, quando distribuídos em grupos por idade (Tabelas 4 e 5).

Quanto ao tempo de leitura por faixa etária, considerando-se tanto a leitura do brinco auricular convencional, quanto a da arruela auricular eletrônica, observou-se que a leitura dos identificadores nos animais do grupo 1 , aqueles com idade até 91 dias, foi mais rápida que nos animais com idade superior a 91 dias $(\mathrm{P}<0,01)$ (Tabela 4). Essa diferença provavelmente ocorre pelo fato de esses animais estarem em fase de aleitamento (aleitamento artificial), o que facilita a contenção e aproximação no momento da leitura, tanto no bezerreiro, para a leitura visual do brinco auricular convencional, quanto no brete, para leitura da arruela auricular eletrônica.

Ao se avaliar o tempo médio gasto com o dispositivo de identificação convencional (brinco auricular), entre os diferentes grupos por idade dos animais (até 91 dias; 92 a 375 dias; mais de 375 dias), foi verificada diferença $(\mathrm{P}<0,05)$ no tempo médio de contenção entre os bovinos mais jovens (até 91 dias; 92 a 375 dias), em relação ao dos mais velhos (mais de 375 dias), assim como no tempo de leitura entre os animais mais jovens (até 91 dias), em relação ao dos animais dos grupos com maior idade (92 a 375 dias; mais de 375 dias). A variável tempo de implantação não apresentou diferenças nas médias de tempo gasto entre os grupos por idade.

Tabela 1. Tempo médio de contenção, de implantação e de leitura do brinco auricular convencional e arruela auricular eletrônica

\begin{tabular}{lcccc}
\hline Variável & Método & $\begin{array}{c}\text { Quantidade } \\
\text { de observações }\end{array}$ & $\begin{array}{c}\text { Tempo médio } \\
\text { (segundos) }\end{array}$ & Valor de $\boldsymbol{P}$ \\
\hline Tempo de contenção & Convencional & 52 & 15,62 & $0,722^{*}$ \\
\hline \multirow{2}{*}{ Tempo de implantação } & Eletrônico & 73 & 15,63 & $0,483^{* *}$ \\
\hline \multirow{2}{*}{ Tempo de leitura } & Convencional & 10 & 47,63 & $0,135^{*}$ \\
\hline
\end{tabular}

${ }^{*}$ Teste de Mann-whitney

${ }^{* *}$ Teste $\mathrm{t}$ de student

Rev. Ceres, Viçosa, v. 60, n.6, p. 757-764, nov/dez, 2013 
As diferenças nessas mesmas variáveis, ao se considerar apenas a arruela eletrônica, não puderam ser avaliadas por haver somente animais com mais de 355 dias de idade no grupo de animais identificados com o dispositivo eletrônico. É importante salientar que, na literatura, não há registros de qual seria o número de observações considerado para análise dessas variáveis. Já, quanto aos animais dos grupos 2 e 3, com mais de 91 dias, que foram contidos no brete, observou-se que não houve diferença significativa na leitura dos diferentes dispositivos de identificação (Tabela 5).

Durante a transferência dos dados do leitor eletrônico para o software não houve erros, sendo gastos para essa ação dois minutos e 42 segundos, para enviar os números dos 23 animais, lidos e armazenados no leitor. Já a digitação de dados manuscritos foi feita por cinco pessoas diferentes, cuja média foi de três minutos e 15 se- gundos para a transcrição e conferência dos números de dez animais, visando à detecção de erros. Vale salientar que os dez animais estavam identificados com números de apenas dois dígitos. Se a quantidade de dígitos fosse maior, haveria maior gasto de tempo e de mão de obra; e maior seria a probabilidade de erros na anotação e transcrição (digitação).

A diferença do tempo gasto para digitar e transferir os dados do leitor foi de 33 segundos (17\%). Essa diferença, do ponto de vista de gestão, é relevante, pois quanto menor o tempo gasto, menor tempo do uso da mão de obra para esse serviço, reduzindo-se o custo. É importante salientar que, ao transferir as informações armazenadas no leitor, independentemente da quantidade de dados contidos no equipamento, o tempo para a transferência desses dados é praticamente o mesmo que o registrado acima; o que poderia haver seria um acréscimo de segun-

Tabela 2. Correlação entre idade e tempo de contenção, de implantação e de leitura em conjunto do brinco auricular convencional e arruela auricular eletrônica

\begin{tabular}{lccc}
\hline Variável & $\begin{array}{c}\text { Quantidade } \\
\text { de observações }\end{array}$ & $\begin{array}{c}\text { Coeficiente } \\
\text { de correlação }\end{array}$ & Valor de $\boldsymbol{P}$ \\
\hline Tempo de contenção & 125 & $-0,261^{*}$ & 0,003 \\
Tempo de implantação & 25 & $-0,129^{* *}$ & 0,537 \\
Tempo de leitura & 208 & $0,256^{*}$ & 0,0001 \\
\hline
\end{tabular}

"Correlação de Spearman; **Correlação de Pearson

Tabela 3. Correlação entre idade e tempo de contenção, de implantação e de leitura separadamente do brinco auricular convencional e arruela auricular eletrônica

\begin{tabular}{lcccc}
\hline Variável & Método & $\begin{array}{c}\text { Quantidade } \\
\text { de observações }\end{array}$ & $\begin{array}{c}\text { Tempo médio } \\
\text { (segundos) }\end{array}$ & Valor de $\boldsymbol{P}$ \\
\hline \multirow{2}{*}{ Tempo de contenção } & Convencional & 52 & $-0,339$ & $0,003^{*}$ \\
& Eletrônico & 73 & $-0,144$ & $0,308^{*}$ \\
\hline \multirow{2}{*}{ Tempo de implantação } & Convencional & 10 & $-0,357$ & $0,191^{* *}$ \\
& Eletrônico & 15 & $-0,046$ & $0,365^{* *}$ \\
\hline \multirow{2}{*}{ Tempo de leitura } & Convencional & 72 & 0,375 & $0,000^{*}$ \\
& Eletrônico & 208 & $-0,186$ & $0,117^{*}$ \\
\hline
\end{tabular}

"Correlação de Spearman;

*** Correlação de Pearson

Tabela 4. Tempo de contenção, de implantação e de leitura em conjunto do brinco auricular convencional e arruela eletrônica em bovinos, de acordo com faixa etária

\begin{tabular}{lcccc}
\hline Variável & Idade (dias) & Observações & Média (segundos) $^{*}$ & Valor de $\boldsymbol{P}$ \\
\hline Tempo de contenção & $\leq 91$ & 21 & 20,63 & $0,153^{* * *}$ \\
& $92-375$ & 44 & 14,54 & 14,67 \\
Tempo de implantação & $>375$ & 60 & 47,92 & $0,617^{* * *}$ \\
& $\leq 91$ & 11 & 46,84 & \\
\hline
\end{tabular}

\footnotetext{
"Valores seguidos por letras diferentes na mesma coluna diferem pelo teste de Mann-whitney

***Teste de ANOVA;

**** Teste de Kruskal-wallis
} 
dos no tempo de transferência, mas a precisão manter-seia. Vale salientar que a maior parte do tempo gasto deveuse a ter -se considerado o tempo necessário para a montagem e preparo do equipamento eletrônico, bem como o da conexão ao microcomputador. Esses tempos foram somados ao tempo de transferência, propriamente dita, e, o total, dividido pela pequena quantidade de animais. À medida que a quantidade de dados a serem "transferidos" aumenta, o tempo de montagem e preparo do equipamento eletrônico será "diluído", contribuindo para aumentar a diferença, justificando a utilização da automação. No caso da transferência manual (digitação), se a quantidade de dados aumentar, a velocidade de digitação diminuirá, bem como aumentará a probabilidade de erros por cansaço do trabalhador.

Uma vantagem da leitura eletrônica está na eliminação de erros de digitação, que ocorreram no caso do brinco auricular convencional, evidenciando a confiabilidade dos dados coletados durante as práticas de manejo e durante a transferência das informações do leitor para o software. Alguns erros de digitação podem ser graves e causar sérias consequências. De acordo com Machado et al. (2000), no processo de identificação animal, deve-se levar em consideração a qualidade e a agilidade no processo de coleta de dados. Na maioria das vezes, essa informação é colhida por funcionários com baixa escolaridade, ou não capacitados. Fazer que as informações sejam registradas corretamente e que sejam devidamente transcritas para o computador, representa dificuldades a serem vencidas pela informatização da propriedade. Outra vantagem da leitura eletrônica é que, segundo Lopes (1997), alguns problemas podem surgir durante a leitura dos brincos convencionais, por desgaste por abrasão dos caracteres e adesão de sujeiras. Quando eles se tornam ilegíveis ou são perdidos, se não estiverem associados a outro método de identificação permanente, o animal perde sua identificação e isso causa prejuízos financeiros e no controle de dados de produção individual.

As despesas com a implantação dos dois métodos (Tabela 6) foram registradas para o cálculo dos custos de implantação. As referentes ao grupo sanidade incluem o unguento e o repelente usados para colocar os dispositivos. Segundo Marsão \& Gonçalves (2009), deve-se tomar os devidos cuidados no momento da aplicação, pois a colocação do brinco causa um ferimento na orelha do animal. Porém, se o dispositivo for aplicado no local correto e ocorrer cicatrização adequada, os riscos de infecção são minimizados e diminuem as chances de perda do brinco. Para o cálculo de mão de obra, considerou-se o tempo gasto para conter os animais, o tempo para implantar cada dispositivo de identificação e o tempo de leitura. Adicionaram-se aos custos os valores referentes ao preço do brinco auricular convencional e ao da arruela auricular eletrônica. Para a análise do custo dos dois métodos, foram registradas todas as despesas para sua implantação e leitura (Tabela 7).

Tabela 5 Tempo de contenção, de implantação e de leitura separadamente do brinco auricular convencional e arruela eletrônica em bovinos, de acordo com a faixa etária

\begin{tabular}{|c|c|c|c|c|}
\hline Variável & Método & Idade (dias) & Observações & Médias (segundos)* \\
\hline \multirow{6}{*}{ Tempo de contenção } & \multirow{3}{*}{ Convencional } & $\leq 91$ & 21 & $15,24^{\mathrm{a}}$ \\
\hline & & $92-375$ & 44 & $8,62^{\mathrm{a}}$ \\
\hline & & $>375$ & 8 & $8,44^{\mathrm{b}}$ \\
\hline & \multirow{3}{*}{ Eletrônico } & $\leq 91$ & 0 & - \\
\hline & & $92-375$ & 0 & - \\
\hline & & $>375$ & 52 & 15,13 \\
\hline \multirow{6}{*}{ Tempo de implantação } & \multirow{3}{*}{ Convencional } & $\leq 91$ & 11 & 48,93 \\
\hline & & $92-375$ & 4 & 46,84 \\
\hline & & $>375$ & 0 & - \\
\hline & \multirow{3}{*}{ Eletrônico } & $\leq 91$ & 0 & - \\
\hline & & $92-375$ & 0 & - \\
\hline & & $>375$ & 10 & 50,47 \\
\hline \multirow{6}{*}{ Tempo de leitura } & \multirow{3}{*}{ Convencional } & $\leq 91$ & 122 & $08,68^{\mathrm{a}}$ \\
\hline & & $92-375$ & 76 & $9,04^{\mathrm{b}}$ \\
\hline & & $>375$ & 10 & $10,92^{\mathrm{b}}$ \\
\hline & \multirow{3}{*}{ Eletrônico } & $\leq 91$ & 0 & - \\
\hline & & $92-375$ & 0 & - \\
\hline & & $>375$ & 72 & 13,56 \\
\hline
\end{tabular}

*Valores seguidos por letras diferentes na mesma coluna diferem pelo teste de Mann-whitney

***Teste de Kruskal-wallis

**** Teste t de student

Rev. Ceres, Viçosa, v. 60, n.6, p. 757-764, nov/dez, 2013 
Para o cálculo do custo fixo, considerou-se, para ambos os métodos, a depreciação do alicate de aplicação. Para o método eletrônico, incluiu-se também o equipamento de leitura. O custo fixo foi responsável por 47,50 e $80,61 \%$ dos custos totais (CT) para os dispositivos de identificação convencional e eletrônico, respectivamente. O equipamento de leitura do dispositivo de identificação eletrônica é responsável pelo elevado custo da depreciação, que poderia ser reduzido pelo aumento da quantidade de animais, pois, de acordo Lopes et al. (2006), a escala de produção influencia significativamente o impacto da depreciação no custo fixo e no custo total, pela otimização da infraestrutura da empresa, até determinados níveis. Os resultados desta pesquisa tornam evidente a necessidade de pesquisas futuras para se estimar a partir de quantos animais esses dispositivos de identificação tornam-se viáveis economicamente, contribuindo com os pecuaristas na tomada de decisão de implantar, ou não, essa tecnologia.

Quanto ao custo operacional efetivo, por animal, o que eleva o custo da arruela auricular eletrônica é o preço de aquisição do dispositivo de identificação, que foi de
$\mathrm{R} \$ 5,42$, ou seja, quatro vezes mais que o do brinco auricular convencional ( $\mathrm{R} \$ 1,30)$. No entanto, a parte da arruela auricular eletrônica em que fica alojado o chip pode ser reutilizada em outro animal, quando o animal identificado for descartado do rebanho. Entretanto, optou-se, neste estudo, por não considerar a reutilização do dispositivo eletrônico no cálculo do custo fixo, o que contribuiu para que o custo fosse maior.

Os dispositivos de identificação, eletrônicos e convencionais, apresentam vantagens e desvantagens. A arruela auricular eletrônica tem como vantagem a agilidade de leitura e de obtenção de dados, bem como a precisão e confiabilidade das informações, e a rapidez na transferência de dados para o computador. Além disso, reduz o risco de fraudes e proporciona o monitoramento do animal por toda a vida útil, evitando que as informações armazenadas durante as práticas de manejo sejam perdidas.

A identificação eletrônica de bovinos poderá ainda ser mais bem aproveitada se associada a balanças eletrônicas e a softwares de gerenciamento e monitoramento de rebanhos, eliminando erros de leitura, de transcrição para planilhas ou fichas individuais e de digitação, reduzindo

Tabela 6. Despesas operacionais efetivas, em reais (R\$) e percentagem (\%), da implantação de brinco auricular convencional e arruela auricular eletrônica por animal

\begin{tabular}{|c|c|c|c|c|}
\hline \multirow{2}{*}{ Especificação } & \multicolumn{2}{|c|}{ Brinco convencional } & \multicolumn{2}{|c|}{ Arruela auricular eletrônica } \\
\hline & $(\mathbf{R} \$)$ & $(\%)$ & $(\mathbf{R} \$)$ & $(\%)$ \\
\hline Mão de obra & 0,05 & 2,60 & 0,05 & 0,83 \\
\hline Sanidade & 0,57 & 29,69 & 0,57 & 9,44 \\
\hline Arruela auricular eletrônica & - & - & 5,42 & 89,74 \\
\hline Brinco auricular convencional & 1,30 & 67,71 & - & - \\
\hline Total por animal & 1,92 & 100,00 & 6,04 & 100,00 \\
\hline
\end{tabular}

Tabela 7. Resumo dos custos dos identificadores convencional e eletrônico, em R \$

\begin{tabular}{lcc}
\hline Especificação & Brinco convencional & Arruela eletrônica \\
\hline Custo operacional total (2+3) & 42,86 & 593,99 \\
Custo total operacional efetivo & 24,96 & 138,79 \\
Custo com depreciação & 17,90 & 455,20 \\
Custo total (5+8) & 48,98 & 737,35 \\
Custos fixos (6+7) & 23,27 & 594,40 \\
Custo com depreciação & 17,90 & 455,20 \\
Remuneração do capital investido & 5,37 & 139,20 \\
Custos variáveis (9+10) & 25,71 & 142,95 \\
Custo operacional efetivo sem impostos & 24,96 & 138,79 \\
Remuneração do capital giro & 0,75 & 4,16 \\
Custo operacional efetivo/animal (2/15) & 1,92 & 6,04 \\
Custo operacional total/animal (1/15) & 3,29 & 25,83 \\
Custo variável médio/animal (8/15) & 1,97 & 6,22 \\
Custo total/animal (4/15) & 3,76 & 32,06 \\
Quantidade de animais identificados (cabeças) & 13,00 & 23,00 \\
Custo fixo em relação ao Custo total (\%) & 47,50 & 80,61 \\
Custo variável em relação ao Custo total (\%) & 52,50 & 18,47 \\
\hline
\end{tabular}


o tempo de tarefas e aumentando a confiabilidade, resultando em maior eficiência do sistema como um todo. Os softwares serão mais ágeis, por causa de sua "alimentação" automática, não sujeita a erros de anotações, e pela possibilidade de oferecerem, aos produtores e técnicos, informações que os auxiliarão na tomada de decisões (Lopes \& Santos, 2007). Como desvantagem, têm custo fixo mais elevado, o que evidência a necessidade da otimização do equipamento, pois esses custos independem da quantidade de animais identificados. As possibilidades de perdas, no caso da arruela eletrônica, ainda existem, semelhantemente às do brinco auricular convencional.

O brinco auricular convencional tem como vantagem menor custo de aquisição, não exigir mão de obra especializada para implantação ou técnica para leitura, ser de fácil identificação visual, ser um dos principais sistemas adotados, ser utilizado em grandes rebanhos, visto que as escalas utilizadas (numérica e alfanumérica) são infinitas. Mas uma de suas desvantagens é ser passível de erros, podendo ter seu número apagado ou ilegível, podendo estar coberto de sujeira, lama, poeira, fatores muito comuns no campo, ocasionando erros de leituras e nas anotações dos dados, não havendo a confiabilidade das informações.

O brinco auricular convencional apresentou menor custo de implantação em relação ao da arruela auricular eletrônica. Além disso, o método convencional não requer equipamento de leitura ou treinamento para sua realização e é acessível, sendo encontrado facilmente no mercado. Mesmo com algumas limitações, como possibilidade de erros de leitura, é um método eficaz na identificação dos animais. O alto custo da arruela eletrônica, por causa do equipamento de leitura, inviabiliza sua implantação em muitas propriedades. Para que a implantação do dispositivo torne-se viável, é necessário avaliar o nível tecnológico e a quantidade de animais da propriedade, a fim de garantir que os custos com o equipamento sejam diluídos com a otimização do uso da mão de obra. Além disso, a arruela auricular eletrônica não é somente um identificador de animais, mas, quando conjugada com outros equipamentos, torna-se ferramenta importante nas rotinas do manejo na propriedade. Com isso, a escolha do método de identificação não deve ser realizada tomando-se como referência apenas o custo financeiro. As vantagens e limitações de cada método devem ser consideradas, pois os dispositivos eletrônicos poderão ser satisfatórios, no que diz respeito à relação custo/benefício, dependendo da quantidade de animais e do sistema de produção.

\section{CONCLUSÕES}

O custo da identificação pelo método da arruela auricular eletrônica foi mais elevado, principalmente em função da aquisição dos equipamentos e dispositivos de identificação. Entretanto, houve maior confiabilidade das informações registradas, já que o brinco auricular convencional, apesar do menor custo, apresentou erros na transcrição dos dados. Assim, cabe a cada produtor e,ou, técnico definir qual deve ser o método de identificação adequado ao seu sistema de produção, considerando não apenas os aspectos financeiros, mas também as limitações e benefícios de cada um.

\section{REFERÊNCIAS}

Banzato E (2002) O paradigma da automação. Disponível em: <http://www.guialog.com.br/ARTIGO345.htm>. Acessado em: 25 de março de 2013.

Curto FPF (2002) Estudo do comportamento de matrizes pesadas (frango de corte), em diferentes ambientes utilizando identificação eletrônica e radio-frequência. Tese de Doutorado. Universidade Estadual de Campinas, Campinas. 134p.

Curto FPF, Marrocco R \& Behrens FH (1997) Sistemas de identificação eletrônica. Revista do Instituto de Informática da PUCCAMP, 5:165-173.

Embrapa - Empresa Brasileira de Pesquisa Agropecuária (1996) Automação de processos. Disponível em: <http:// www.cnpdia.embrapa.br/pesquisa/auto.html>. Acessado em: 25 de março de 2013.

Hoffmann R, Engler JJC, Serrano O, Thame ACM \& Neves EM (1981) Administração da empresa agrícola. 3a ed. São Paulo, Pioneira. 325p.

Jardim VR (1973) Curso de bovinocultura. 4 ${ }^{\mathrm{a}}$ ed. Campinas, Instituto Campineiro de Ensino Agrícola. 526p.

Lopes MA \& Santos G (2007) Custo da implantação da rastreabilidade bovina e bubalina utilizando os diferentes métodos de identificação permitidos pelo SISBOV. Ciência Animal Brasileira, 8:657-664.

Lopes MA (1997) Informática aplicada à bovinocultura . Jaboticabal, FUNEP. 82p.

Lopes MA (2005) Rastreabilidade na bovinocultura. 2a ed. Lavras, FAEPE/PROEX. 76p.

Lopes MA (2003) Zootecnia de precisão. Lavras, FAEPE/PROEX. $135 \mathrm{p}$.

Lopes MA \& Vieira PF (1998) Criação de bezerros leiteiros. Jaboticabal, FUNEP. 69p.

Lopes MA, Carmo EA, Lima ALR \& Carvalho FM (2006) Análise da rentabilidade de uma empresa com opção de comercialização de queijo e leite. Arquivo Brasileiro de Medicina Veterinária e Zootecnia, 58:642-647.

Lopes MA, Santos G \& Amado GB (2008) Viabilidade econômica da adoção e implantação da rastreabilidade em sistemas de produção de bovinos no Estado de Minas Gerais. Ciência e Agrotecnologia, 2:288-294.

Machado JGCF, Nantes JFD \& Machado CGCF (2000) Avaliação de um sistema de identificação eletrônica de animais na rastreabilidade de informações. Revista Brasileira de Agrocomputação, 1:13-21.

Marsão DJM \& Gonçalves AC (2009) Sistemas de identificação de ovinos: PROJEPEC. Disponível em: <www.projepec.com.br/ Sistemasdeidentificacaodeovinos.pdf $>$. Acessado em: 20 de agosto de 2009.

SPSS Inc. (2011) SPSS Base 17.0 para o Windows. Guia do Usuário. Chicago, SPSS Inc. $639 \mathrm{p}$. 